\title{
Overview of the interpretation of indirect searches for charged Higgs bosons in the 2HDM
}

\author{
Farvah Mahmoudi*† \\ Univ Lyon, Univ Lyon 1, ENS de Lyon, CNRS, Centre de Recherche Astrophysique de Lyon \\ UMR5574, F-69230 Saint-Genis-Laval, France \\ CERN, Theoretical Physics Department, CH-1211 Geneva 23, Switzerland \\ E-mail: nazila@cern.ch
}

The implications of indirect searches through rare $B$ decays is presented in Two Higgs Doublet Models, with an emphasis on the recent $\mathrm{LHCb}$ measurements. A comparison with the direct LHC search constraints is also provided. In particular, in 2HDM of Type II, we find that flavour physics observables impose a combined lower limit on the charged Higgs mass of $M_{H^{ \pm}} \gtrsim 600$ $\mathrm{GeV}$ - independent of $\tan \beta$ - which increases to $M_{H^{ \pm}} \gtrsim 650 \mathrm{GeV}$ for $\tan \beta<1$.

Prospects for Charged Higgs Discovery at Colliders

3-6 October 2016

Uppsala, Sweden

\footnotetext{
* Speaker.

${ }^{\dagger}$ Also Institut Universitaire de France, 103 boulevard Saint-Michel, 75005 Paris, France.
} 


\section{Introduction}

While the main objective of flavour physics experiments in the past was the tests of the Standard Model (SM) and the CKM paradigm, the focus is now towards the new physics (NP) and search for the indirect signs of new particles. Indeed, low-energy flavour-changing processes are sensitive to energies scales that are much higher than energies accessible at the colliders. Collider searches provide direct information on the mass spectrum of the possible new particles. On the other hand, the indirect information from flavour observables provide constraints on their couplings. The two sectors are therefore nicely complementary.

Here we consider the Two-Higgs-Doublet-Model (2HDM), a well motivated class of models obtained by extending the SM Higgs sector by a second scalar $\mathrm{SU}(2)_{L}$ doublet, which leads to three neutral Higgs bosons and a charged Higgs boson, and we focus on rare $B$ decays and the implication of the recent measurements at the LHCb experiment in the context of $Z_{2}$ symmetric scenarios of $2 \mathrm{HDM}$. We study the flavour physics constraints on the charged Higgs mass and the complementarity with direct searches of heavy Higgs bosons at LEP and the LHC.

The work presented here is an extension and update of the analysis in [1]. More details can be found in [2].

\section{Flavour constraints}

For the present analysis we have chosen a set of "conventional" flavour physics observables sensitive to charged Higgs exchange. These are listed in Table 1 where we give the experimentally measured values and the corresponding SM predictions. In addition, we consider the recent measurements in the $B \rightarrow K^{(*)} \ell^{+} \ell^{-}$and $B_{s} \rightarrow \phi \mu^{+} \mu^{-}$decays, where LHCb has measured a multitude of clean angular observables in addition to the branching ratios. These measurements are performed in bins of the dilepton invariant mass, $q^{2}$. Most of these observables are in good agreement with the SM predictions. However, some deviations from the SM predictions were observed in certain $q^{2}$ bins for some of the observables. The largest deviation with $\sim 3 \sigma$ significance was reported in the $q^{2} \in[4.0,6.0]$ and $[6.0,8.0] \mathrm{GeV}^{2}$ bins for the angular observable $P_{5}^{\prime}$ [6]. Moreover, the branching ratio of the decay $B_{s} \rightarrow \phi \mu^{+} \mu^{-}$is found to be

\begin{tabular}{|l|l|l|}
\hline Observable & Experiment & SM prediction \\
\hline $\mathrm{BR}\left(B \rightarrow X_{s} \gamma\right)$ & $(3.32 \pm 0.15) \times 10^{-4}[3]$ & $(3.34 \pm 0.22) \times 10^{-4}$ \\
\hline$\Delta_{0}\left(B \rightarrow K^{*} \gamma\right)$ & $(1.2 \pm 5.1) \times 10^{-2}[3]$ & $(5.33 \pm 2.6) \times 10^{-2}$ \\
\hline $\mathrm{BR}\left(B_{s} \rightarrow \mu^{+} \mu^{-}\right)$ & $(3.0 \pm 0.6 \pm 0.25) \times 10^{-9}[4]$ & $(3.54 \pm 0.27) \times 10^{-9}$ \\
\hline $\mathrm{BR}\left(B_{u} \rightarrow \tau v\right)$ & $(1.06 \pm 0.19) \times 10^{-4}[3]$ & $(0.82 \pm 0.29) \times 10^{-4}$ \\
\hline $\mathrm{BR}\left(D_{s} \rightarrow \tau v\right)$ & $(5.51 \pm 0.24) \times 10^{-2}[3]$ & $(5.13 \pm 0.11) \times 10^{-2}$ \\
\hline$\Delta M_{B_{s}}$ & $17.76 \pm 0.021 \mathrm{ps}^{-1}[3]$ & $17.38 \pm 1.505 \mathrm{ps}^{-1}$ \\
\hline
\end{tabular}

Table 1: Experimental results and the corresponding SM values for a selected number of flavour physics observables. The experimental data represents the most recent measurements or official combinations. The experimental values and SM predictions for the observables related to $B \rightarrow K^{(*)} \ell^{+} \ell^{-}$and $B_{s} \rightarrow \phi \mu^{+} \mu^{-}$can be found in Ref. [5]. 
more than $3 \sigma$ below the SM predictions [7]. LHCb has in addition performed tests of lepton flavour universality by measuring the ratios $R_{K}=\mathrm{BR}\left(B^{+} \rightarrow K^{+} \mu^{+} \mu^{-}\right) / \mathrm{BR}\left(B^{+} \rightarrow K^{+} e^{+} e^{-}\right)$and $R_{K^{*}}=\mathrm{BR}\left(B \rightarrow K^{* 0} \mu^{+} \mu^{-}\right) / \mathrm{BR}\left(B \rightarrow K^{* 0} e^{+} e^{-}\right)$in the low- $q^{2}$ region showing $2.6 \sigma$ and $\sim 2.5 \sigma$ deviations respectively $[8,9]$. In this study, we include in a consistent way all these observables by performing a statistical combination taking into account both the theoretical and experimental correlations. The methodology and the list of observables with their definitions follow closely Refs. [5, 10,11].

Other deviations from the SM predictions have been reported by the Belle and BaBar experiments in the measurements of the ratios $\mathrm{BR}(B \rightarrow D \tau v) / \mathrm{BR}(B \rightarrow D \ell v)$ and $\mathrm{BR}\left(B \rightarrow D^{*} \tau v\right) / \mathrm{BR}(B \rightarrow$ $D^{*} \ell v$ ) (where $\ell$ is $e$ or $\mu$ ) that exceed the SM expectations by $\sim 4 \sigma$ [3]. As the hadronic form factors tend to cancel in these ratios, they are rather sensitive to new physics contributions. A consistent explanation of the two ratios is not possible neither in the 2HDM Type II nor in the MSSM. On the other hand, the recent Belle results [12] are in agreement with the SM predictions within $0.6 \sigma$ level. Therefore, we do not include them in the global fits of the flavour observables.

For the numerical evaluation of the SM predictions we use Super I so (version 3.7) [13-15], which is also used for the evaluation of the 2HDM contributions.

\section{New Physics interpretation}

We first study the constraining power of the recent $b \rightarrow s \ell \ell$ data in a model independent way using a global fit within the minimal flavour violation (MFV) framework. The constraints for the relevant Wilson coefficients are presented in Figure 1. Here in addition to the $b \rightarrow s \ell \ell$ data, the constraints from $\mathrm{BR}\left(B \rightarrow X_{s} \gamma\right)$ and the isospin asymmetry of $B \rightarrow K^{*} \gamma$ are also considered (see Ref. [5] for details). Within the MFV paradigm, the SM can accommodate the current data at the $2-\sigma$ level.
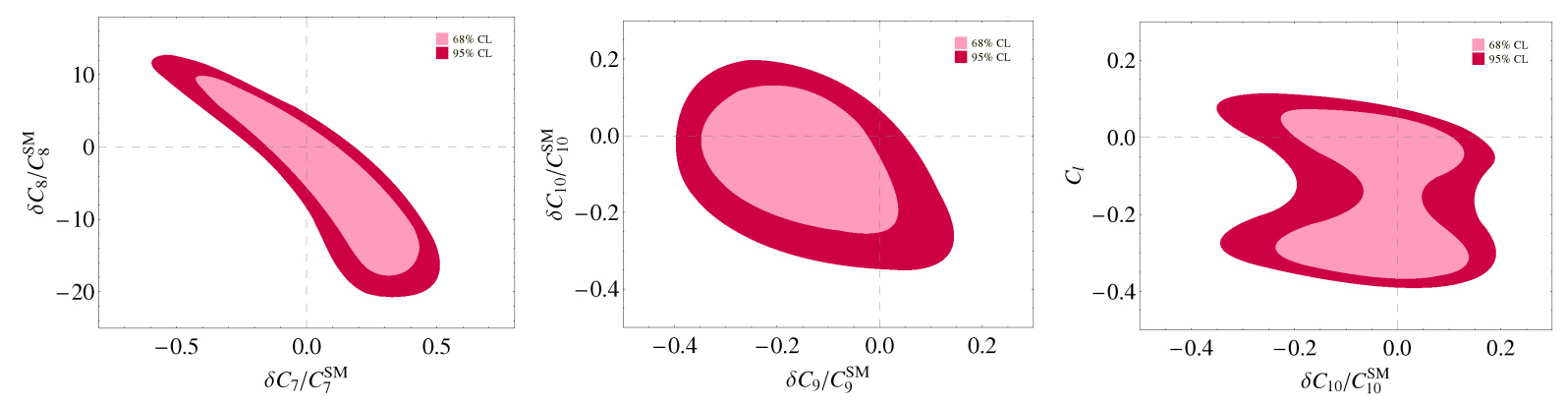

Figure 1: Global fit results for new physics contributions to the Wilson coefficients $\delta C_{i}$ normailised by their SM values.

\section{Implications in Two-Higgs Doublet Models}

Next we consider the constraints in the Two-Higgs Doublet Models. Phenomenologically, the $2 \mathrm{HDM}$ is a very useful and widely studied extension of the SM Higgs sector. It exhibits a plethora 


\begin{tabular}{cccc}
\hline Type & $\lambda_{U U}$ & $\lambda_{D D}$ & $\lambda_{L L}$ \\
\hline I & $\cot \beta$ & $\cot \beta$ & $\cot \beta$ \\
II & $\cot \beta$ & $-\tan \beta$ & $-\tan \beta$ \\
III & $\cot \beta$ & $-\tan \beta$ & $\cot \beta$ \\
IV & $\cot \beta$ & $\cot \beta$ & $-\tan \beta$ \\
\hline
\end{tabular}

Table 2: Yukawa couplings for the four types of 2 HDM. $U, D$ and $L$ stand respectively for the up-type quarks, the down-type quarks and the leptons.

of collider signatures for LHC BSM Higgs searches and constitutes a useful benchmark to study the impact of flavour physics data, as we will discuss below.

If we restrict ourselves to the case with a $Z_{2}$ symmetry to prevent tree-level flavour changing neutral currents (FCNC), only one doublet couples to each class of fermions. There are four physically distinct $Z_{2}$ charge assignments, which are labelled as 2HDM Types I-IV [16]. Our conventions for the types are listed in Table 2.

The couplings of the charged Higgs boson to fermions, which will be the most important for our studies, are given by $-i g_{H^{+} \bar{f} f^{\prime}}$, with, for quarks,

$$
g_{H^{+} \bar{U} D}=\frac{V_{U D}}{\sqrt{2} M_{W}}\left[\lambda_{U U} \frac{1-\gamma_{5}}{2}+\lambda_{D D} \frac{1+\gamma_{5}}{2}\right]
$$

and for leptons

$$
g_{H^{+} \bar{v}_{L} L}=\frac{1}{\sqrt{2} M_{W}} \lambda_{L L} \frac{1+\gamma_{5}}{2} .
$$

The most relevant Wilson coefficients for $b \rightarrow s$ transitions are the electromagnetic, chromomagnetic, and vector and axial electroweak ones, which receives at the electroweak scale the following contributions in the $2 \mathrm{HDM}$, respectively:

$$
\delta C_{7,8}^{H^{ \pm}(0)}=\frac{1}{3} \lambda_{t t}^{2} F_{7,8}^{(1)}(y)-\lambda_{t t} \lambda_{b b} F_{7,8}^{(2)}(y)
$$

where $y=\bar{m}_{t}^{2} / m_{H^{+}}^{2}$ and $F_{7,8}^{(1,2)}$ are positive functions, and

$$
\begin{aligned}
& \delta C_{9}^{H^{ \pm}(0)}=\frac{1-4 s_{W}^{2}}{s_{W}^{2}} \mathscr{C}^{H^{ \pm}(0)}\left(x_{t H^{ \pm}}\right)-\mathscr{D}^{H^{ \pm}(0)}\left(x_{t H^{ \pm}}\right), \\
& \delta C_{10}^{H^{ \pm}(0)}=-\frac{1}{s_{W}^{2}} \mathscr{C}^{H^{ \pm}(0)}\left(x_{t H^{ \pm}}\right),
\end{aligned}
$$

with $x_{t H^{ \pm}}=\frac{m_{t}^{2}}{M_{H^{ \pm}}^{2}}$ and

$$
\begin{aligned}
& \mathscr{C}^{H^{ \pm}(0)}(x)=\frac{M_{H^{ \pm}}^{2}}{8 M_{W}^{2}} \lambda_{t t}^{2} x^{2}\left\{\frac{-1}{(x-1)^{2}} \ln x+\frac{1}{x-1}\right\} \\
& \mathscr{D}^{H^{ \pm}(0)}(x)=\frac{1}{18} \lambda_{t t}^{2} x\left\{\frac{-3 x^{3}+6 x-4}{(x-1)^{4}} \ln x+\frac{47 x^{2}-79 x+38}{6(x-1)^{3}}\right\} .
\end{aligned}
$$



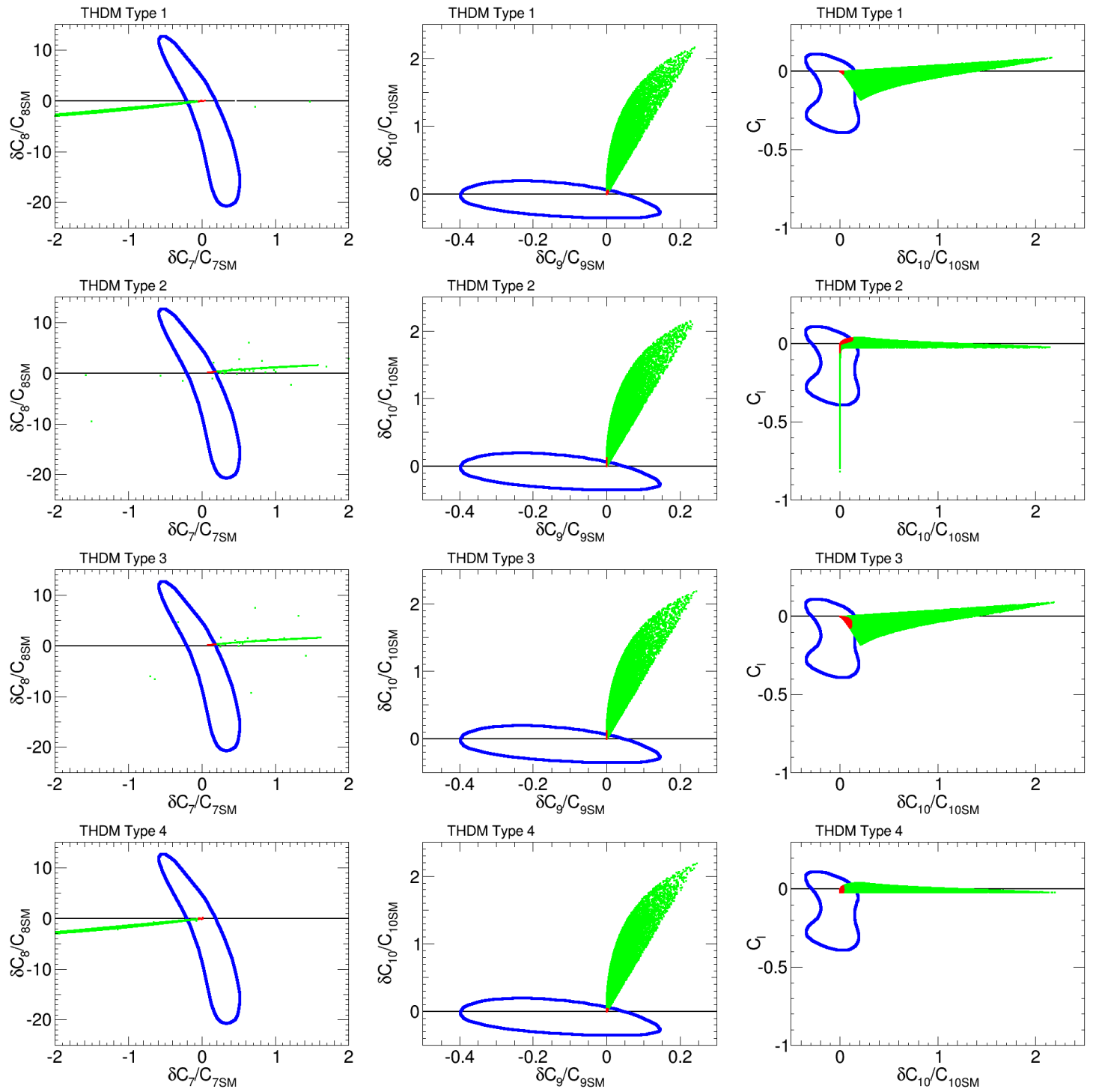

Figure 2: Values of the 2HDM contributions to the Wilson coefficients normalised to the SM ones, compared to the $95 \%$ C.L. regions obtained through MFV model-independent fits of the $b \rightarrow s$ transitions, for the four types. The green regions encompass all our 2HDM points, and the small red zones include the points in agreement at $95 \%$ C.L. with the model-independent fit.

$C_{7,8}$ contains two terms, one proportional to $\lambda_{t t}^{2}$, i.e. $1 / \tan ^{2} \beta$ for all four types, and the other to $-\lambda_{t t} \lambda_{b b}$, i.e. +1 for types II and III and $-1 / \tan ^{2} \beta$ for types I and IV. In types II and III, these contributions add constructively to the SM Wilson coefficients. For $\delta C_{9,10}$, the LO contributions are proportional to $\lambda_{t t}^{2}$, i.e. $1 / \tan ^{2} \beta$ for all four types, and are always of the same sign as $C_{9,10}^{\mathrm{SM}}$.

In Fig. 2, a comparison of the values of the relevant Wilson coefficients in the 2HDM with the MFV model-independent analysis results of Section 3 is shown. We see that most of the 2HDM points are not in agreement with the model-independent fit results, and only a very small region is selected.

The combined flavour constraints are shown in Figs. 3 and 4, where the different colours show 

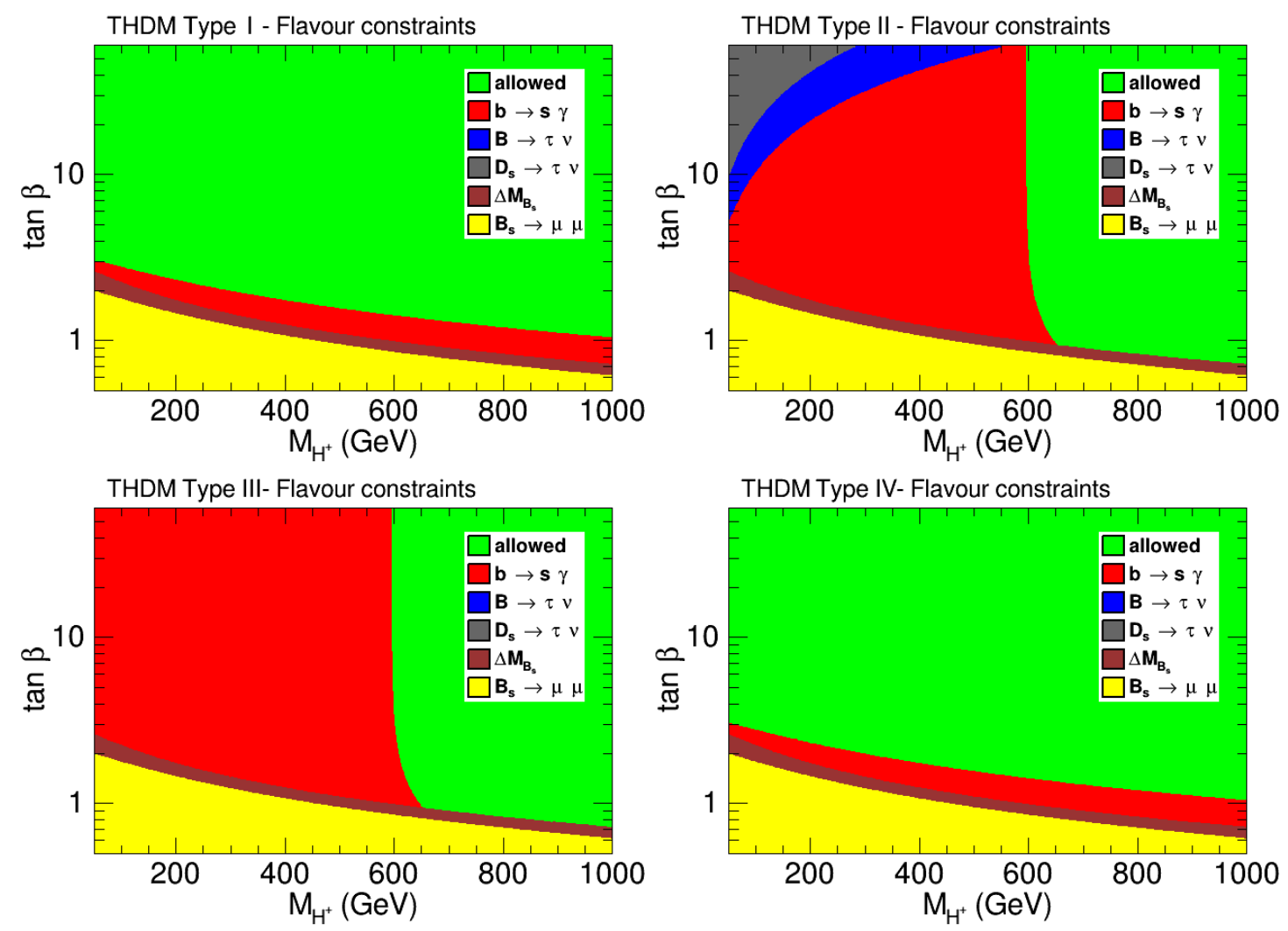

Figure 3: Regions of the $\left(M_{H^{+}}, \tan \beta\right)$ parameter space indirectly excluded by flavour constraints at $95 \%$ C.L. for the different 2HDM Yukawa types. The colour coding corresponds to exclusion by different constraints, as described by the legend. The green region is allowed by all constraints.

exclusion (at 95\% C.L.) by different flavour observables (as indicated by the legend), for the four types in the $\left(M_{H^{+}}, \tan \beta\right)$ parameter plane. The constraints in Fig. 4 have been obtained by means of the minimisation of the $\chi^{2}$ for the different $b \rightarrow s$ transitions, considering the experimental and theoretical correlations and following the method presented in [11]. In the general 2HDM (unlike the supersymmetric case) the results presented in the figures have a negligible dependence on other parameters of the model. In type II and III models, a $\tan \beta$-independent lower limit of $M_{H^{ \pm}} \gtrsim 600$ $\mathrm{GeV}$ is obtained from the $\mathrm{BR}\left(B \rightarrow X_{s} \gamma\right)$ constraint.

For all types, we find that values of $\tan \beta<1$ are ruled out for all values of $M_{H^{ \pm}}<650 \mathrm{GeV}$, including the important non-decoupling region of the type I model. There are several constraints which are mainly sensitive to the type II model at high $\tan \beta$, such as the $\operatorname{BR}\left(B \rightarrow \tau \nu_{\tau}\right)$.

We then consider the Higgs searches at LEP and the LHC. The couplings of the Higgs bosons are calculated using 2HDMC (version 1.7.0) [17], and the LEP and LHC limits are obtained with HiggsBounds (version 5.1.0beta) [18-21]. In Fig. 5, the results are shown for the MSSM-like scenario with $M_{H}=M_{A}=M_{H^{+}}$. Contrary to the flavour constraints, the Higgs limits depend on the choice of the other parameters, and in particular on the neutral Higgs masses. More detail can be found in [2]. The LEP and LHC charged Higgs constraints are relatively robust, but the neutral Higgs searches can lead to different results for other choices of $M_{H}$ and $M_{A}$. For types I and IV, 

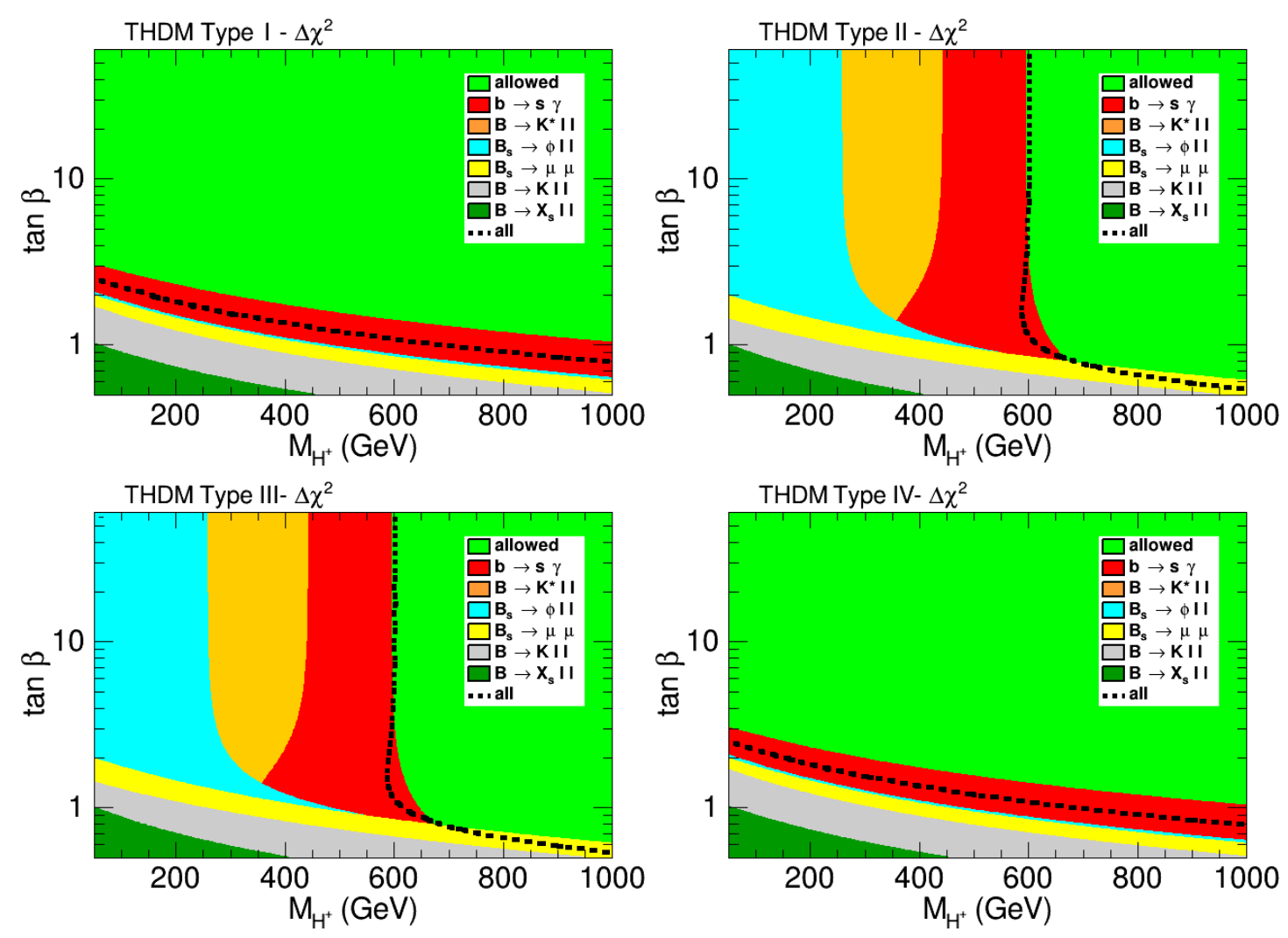

Figure 4: Regions of the $\left(M_{H^{+}}, \tan \beta\right)$ parameter space indirectly excluded by flavour constraints at $95 \%$ C.L. for the different 2HDM Yukawa types. The colour coding corresponds to exclusion by different constraints, as described by the legend. The green region is allowed by all constraints.

flavour physics and direct searches provide complementary constraints, flavour physics excludes small $\tan \beta$ regions, while collider searches can also probe large $\tan \beta$. In types II and IV, flavour observables set very strong constraints, and only specific neutral Higgs search channels can go beyond them in the large $\tan \beta$ region.

\section{Conclusions}

We studied the constraints from flavour physics, and in particular from the $b \rightarrow s$ transitions, on the parameter space of the $2 \mathrm{HDM}$ scenarios with $Z_{2}$ symmetry. We showed that flavour physics observables provide very strong constraints on the charged Higgs sector, which depend (to a very good approximation) only on $M_{H^{+}}$and $\tan \beta$. In particular, the inclusive decay $b \rightarrow s \gamma$ and, more generally, the $b \rightarrow s$ transitions lead to a robust exclusion of charged Higgs masses below $600 \mathrm{GeV}$ for types II and III, and of $650 \mathrm{GeV}$ for $\tan \beta<1$ for all Yukawa types.

Considering the LHC Higgs searches, we showed that, whereas limits from direct charged Higgs searches at LEP and the LHC are very robust with respect to the variation of the 2HDM parameters for each of the four types, the constraints from neutral Higgs searches at the LHC strongly depend on the choice of parameters, and thus do not provide model-independent limits on the charged Higgs mass. Nevertheless, in the MSSM-like scenario where all heavy Higgs bosons 

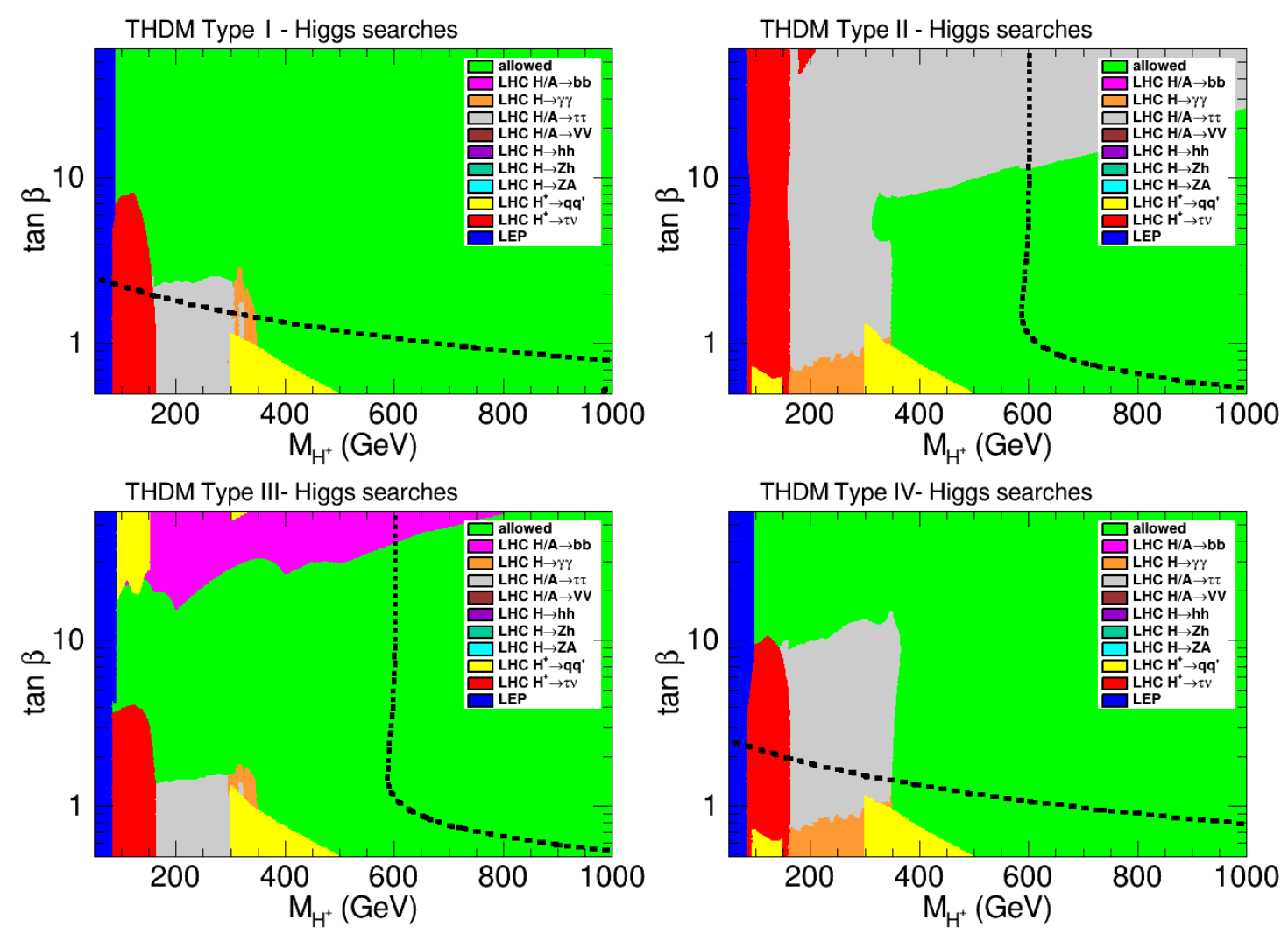

Figure 5: Regions of the $\left(M_{H^{+}}, \tan \beta\right)$ parameter space indirectly excluded by flavour constraints at $95 \%$ C.L. for the different 2HDM Yukawa types. The colour coding corresponds to exclusion by different constraints, as described by the legend. The green region is allowed by all constraints.

have equal masses, we find that neutral Higgs searches do provide important constraints, and complement the constraints from direct charged Higgs searches and flavour physics.

\section{Acknowledgements}

The author would like to thank the organisers for their invitation and for the very fruitful workshop, and is grateful to O. Stål with whom this work was initiated and to A. Arbey and T. Stefaniak for their collaboration in updating this analysis.

\section{References}

[1] F. Mahmoudi and O. Stål, Phys. Rev. D 81 (2010) 035016 [arXiv:0907.1791 [hep-ph]].

[2] A. Arbey, F. Mahmoudi, O. Stål and T. Stefaniak, arXiv:1706.07414 [hep-ph].

[3] Y. Amhis et al., arXiv:1612.07233 [hep-ex].

[4] R. Aaij et al. [LHCb Collaboration], Phys. Rev. Lett. 118 (2017) no.19, 191801 [arXiv:1703.05747 [hep-ex]].

[5] T. Hurth, F. Mahmoudi and S. Neshatpour, Nucl. Phys. B 909 (2016) 737 [arXiv:1603.00865 [hep-ph]]. 
[6] R. Aaij et al. [LHCb Collaboration], JHEP 1602 (2016) 104 [arXiv:1512.04442 [hep-ex]].

[7] R. Aaij et al. [LHCb Collaboration], JHEP 1509 (2015) 179 [arXiv:1506.08777 [hep-ex]].

[8] R. Aaij et al. [LHCb Collaboration], Phys. Rev. Lett. 113 (2014) 151601 [arXiv:1406.6482 [hep-ex]].

[9] R. Aaij et al. [LHCb Collaboration], arXiv:1705.05802 [hep-ex].

[10] V. G. Chobanova, T. Hurth, F. Mahmoudi, D. Martinez Santos and S. Neshatpour, arXiv:1702.02234 [hep-ph].

[11] T. Hurth, F. Mahmoudi, D. Martinez Santos and S. Neshatpour, arXiv:1705.06274 [hep-ph].

[12] A. Abdesselam et al., arXiv:1608.06391 [hep-ex].

[13] F. Mahmoudi, Comput. Phys. Commun. 178 (2008) 745 [arXiv:0710.2067 [hep-ph]].

[14] F. Mahmoudi, Comput. Phys. Commun. 180 (2009) 1579 [arXiv:0808.3144 [hep-ph]].

[15] F. Mahmoudi, Comput. Phys. Commun. 180 (2009) 1718.

[16] V. D. Barger, J. L. Hewett and R. J. N. Phillips, Phys. Rev. D 41 (1990) 3421.

[17] D. Eriksson, J. Rathsman and O. Stal, Comput. Phys. Commun. 181 (2010) 189 [arXiv:0902.0851 [hep-ph]].

[18] P. Bechtle, O. Brein, S. Heinemeyer, G. Weiglein and K. E. Williams, Comput. Phys. Commun. 181 (2010) 138 [arXiv:0811.4169 [hep-ph]].

[19] P. Bechtle, O. Brein, S. Heinemeyer, G. Weiglein and K. E. Williams, Comput. Phys. Commun. 182 (2011) 2605 [arXiv:1102.1898 [hep-ph]].

[20] P. Bechtle, O. Brein, S. Heinemeyer, O. Stål, T. Stefaniak, G. Weiglein and K. E. Williams, Eur. Phys. J. C 74 (2014) no.3, 2693 [arXiv:1311.0055 [hep-ph]].

[21] P. Bechtle, S. Heinemeyer, O. Stål, T. Stefaniak and G. Weiglein, Eur. Phys. J. C 75 (2015) no.9, 421 [arXiv:1507.06706 [hep-ph]]. 